\title{
Bilateral xanthogranulomatous inflammation of nasal and nasopharynx mass: a case report
}

Wei Jie Boon ${ }^{1}$, Khim Soon Vong $^{1 *}$ and Ing Ping Tang ${ }^{1,2^{*}}$

\begin{abstract}
Background: Xanthogranulomatous inflammation is an uncommon condition in adult nasal cavity and nasopharynx.

Case presentation: We report a male patient who developed xanthogranulomatous inflammation in the bilateral nose and nasopharynx. The patient was not responsive to oral steroid. Multiple biopsies were taken to confirm the definitive diagnosis, and endoscopic debulking of mass was performed to provide symptomatic relief. Patient was free from nasal symptoms, and residual nasal and nasopharyngeal masses showed no increment in size and number during follow-up.

Conclusion: Xanthogranulomatous inflammation in the nasal cavity and nasopharynx can be easily confused with other nasal masses. A histopathology examination is crucial to establish the diagnosis. A repeated biopsy is needed if previous biopsy result is inconclusive. Surgical excision is recommended for symptomatic relief. The effectiveness of medical treatment is still unclear. A long-term follow-up is mandatory.
\end{abstract}

Keywords: Xanthogranulomatous inflammation, Nasal cavity mass, Nasopharynx mass

\section{Background}

Xanthogranulomatous inflammation is a rare chronic inflammatory condition that is characterized by aggregation of the lipid-laden foamy macrophages or xanthoma cells. This disease entity is well recognised in the kidney and gallbladder $[1,2]$. It is seldom detected in the nasal cavity and nasopharynx. Due to its rarity and non-specific presentation, xanthogranulomatous inflammation can be confused with other benign/malignant tumour preliminary. Thus, we report a case of xanthogranulomatous inflammation in bilateral nasal cavity and nasopharynx in a 50-year-old gentleman and highlight its diagnostic challenges.

\footnotetext{
*Correspondence: ahsoonv@yahoo.com; ingptang@yahoo.com

${ }^{1}$ Department of ORL HNS, Sarawak General Hospital, Kuching, Malaysia

2 Department of ORL HNS, Faculty of Medicine and Health Sciences,

Universiti Malaysia Sarawak, Kota Samarahan, Malaysia
}

\section{Case presentation}

A 50-year-old male health care worker with underlying hypertension and diabetes mellitus type II presented with epistaxis, nasal block and hyposmia for the past 4 months. Endoscopic examination showed firm irregular lobulated yellowish-reddish masses arising from the right anterior $1 / 3$ of the septal nasal mucosa and left lateral wall. Bilateral torus tubarius appeared as a hypertrophy with irregular yellowish lesion on each medial surface (Fig. 1).

Cutaneous examination revealed mobile, firm, papulonodular lesion at different sites of his body (Fig. 2). The first histopathological result of the nasal and nasopharynx biopsies reviewed granulomatous inflammation. Relevant laboratory investigations showed negative results for tuberculosis, Wegener granulomatosis and lymphoma. Radiographic examination was performed to characterize the composition and extension of the mass (Fig. 3). 


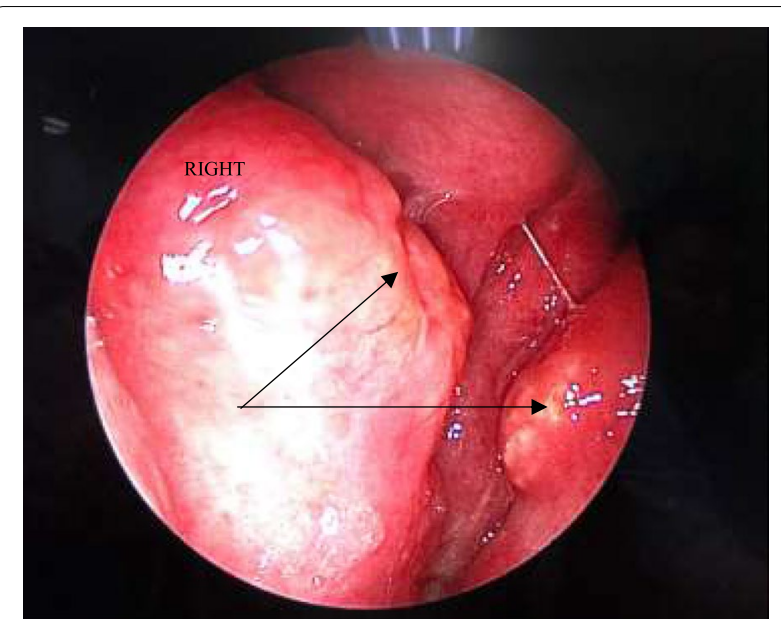

Fig. 1 Endoscopic view of nasopharynx showed the right torus tubarius is more prominent than the left side with irregular yellowish lobulated lesion on the medial surface

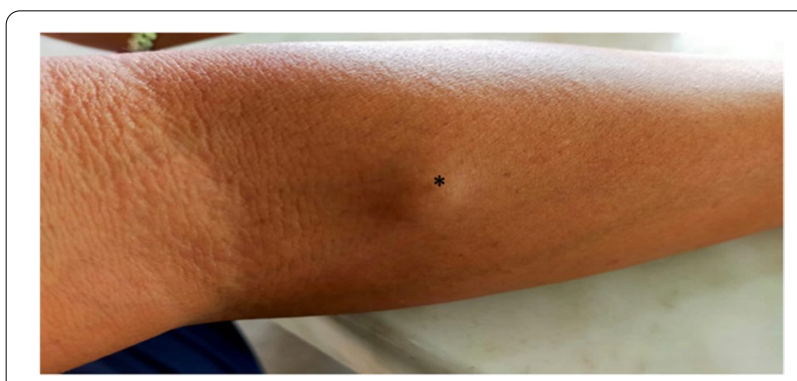

Fig. 2 View of papulonodular lesion at forearm $\left(^{*}\right)$
Repeated nasal biopsies revealed xanthogranulomatous inflammation. Oral prednisolone was prescribed to the patient empirically with $50 \mathrm{mg}$ OD with tapering dose for a total of 1 month and continued with maintenance dose of $20 \mathrm{mg}$ OD for another 1 month; however, the nasal symptoms were not improved, and the masses were not reduced in size. Endoscopic transnasal excision and debulking of bilateral nasal masses was performed subsequently. Intraoperatively, the operative sites were noted to ooze actively which causes difficulty in excising the masses completely. At post-operative 6 months, despite the presence of residual nasal mass, the patient is asymptomatic without any recurrent epistaxis and nasal blockage (Fig. 4).

\section{Discussion}

Xanthogranulomatous (XG) processes are rare inflammatory conditions with the characteristic pathologic feature of lipid-laden macrophages or histiocyte cells. Infection and inflammation, histiocytic processes or inherited lysosomal disorders could be the causes of xanthogranulomatous process. Pyelonephritis and cholecystitis are commonly seen in infectious or inflammatory type processes of XG. Histiocytosis is grouped into Langerhans and non-Langerhans cell histiocytosis. Summarise classification of XG processes is showed in Fig. 5. Pathophysiologically, a great number of neutrophil, lymphocyte, plasma cells, fibroblasts, foreign body giant cells and histiocyte are seen in the acute phase of the infectious/inflammatory XG process, which result in capillary proliferation, collagen formation and haemorrhage. Xanthoma cell is formed when the histiocytes engulf the erythrocytes. The consequence of the whole inflammatory process is the formation of granulation and necrosis debris. Eventually, the organ tissue is replaced by xanthoma cells, formation of granulomas, and proliferation

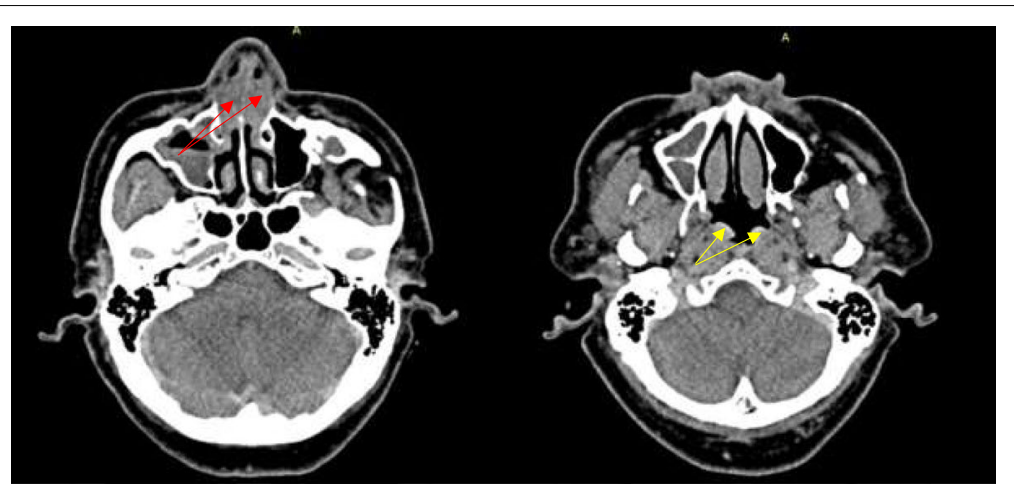

Fig. 3 Axial contrast-enhanced CT images showing enhancing masses at bilateral anterior nasal cavities (red arrows) and nasopharynx (yellow arrows) 

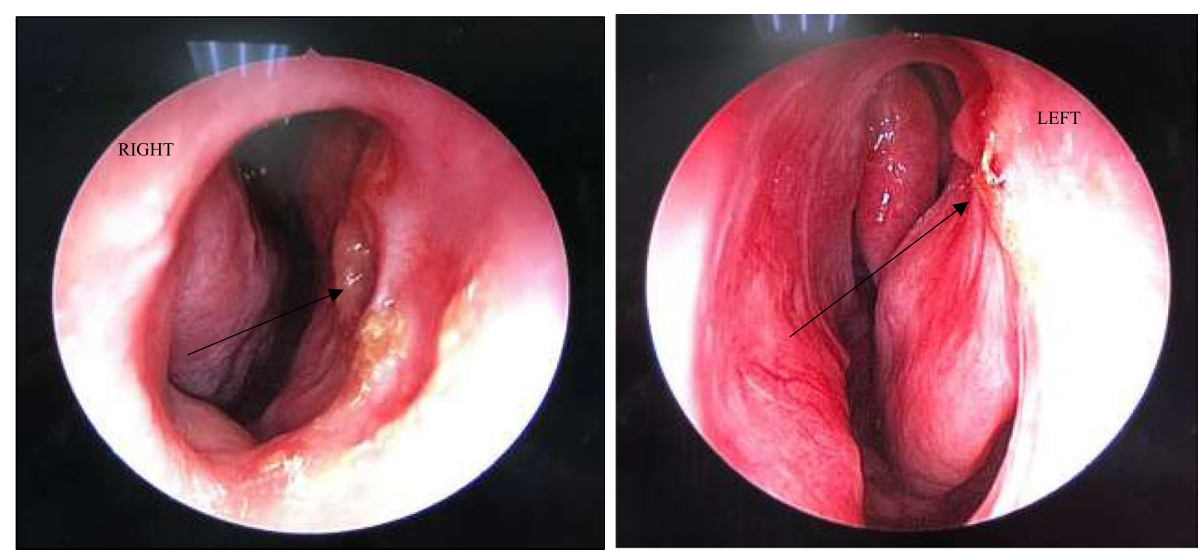

Fig. 4 Endoscopic examination post operatively showed a remnant of xanthogranuloma at the right nasal septum and left lateral nasal wall

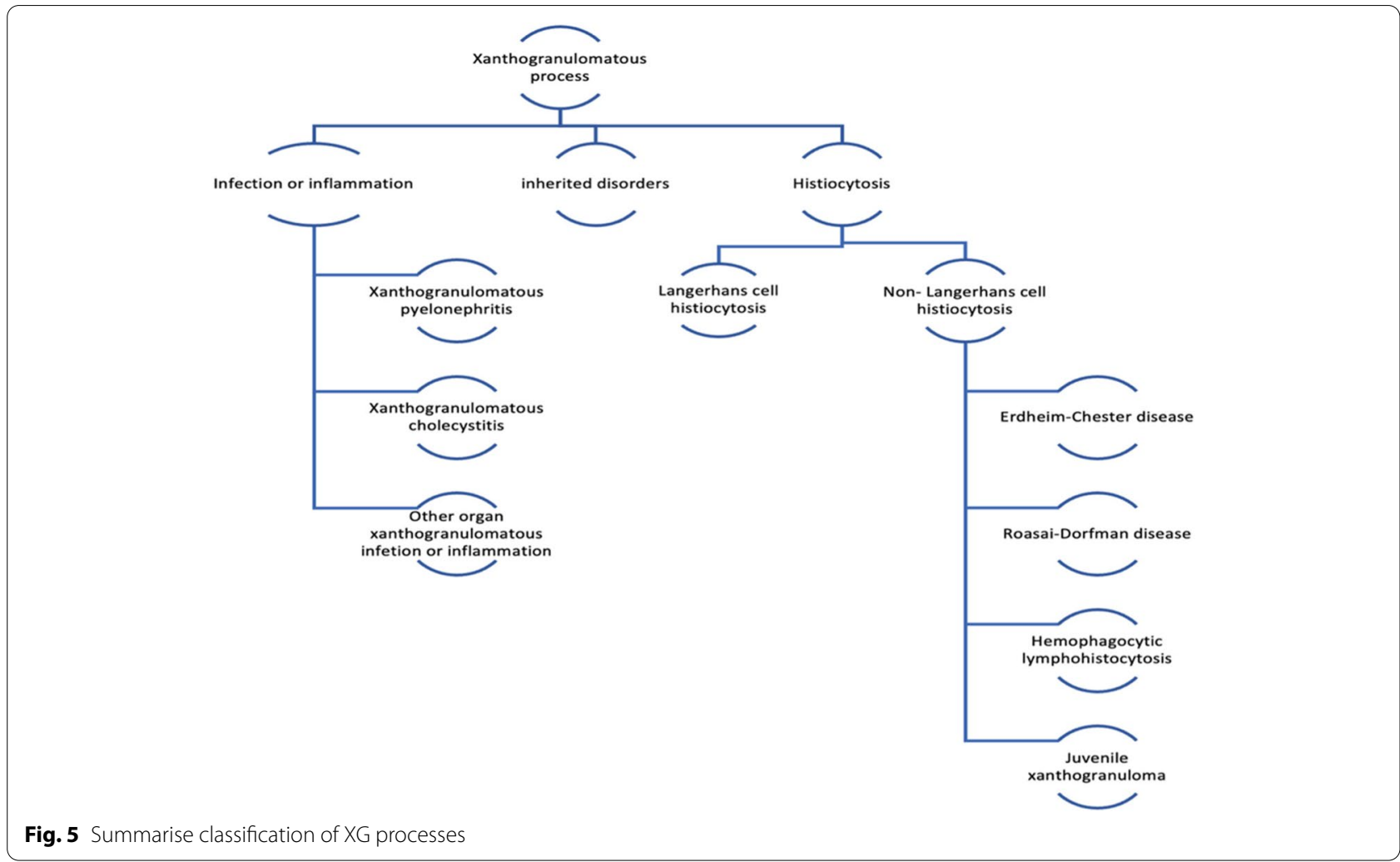

of fibrin and collagen deposition, giving rise to tissue destruction [1].

Xanthogranulomatous inflammation in the nasal cavity and nasopharynx can mimic other nasal masses which makes it represent a challenging diagnostic problem. The differential diagnosis range from benign to highly malignant conditions which compose of inflammatory (rhinosinusitis with polyp, Samter's triad), infection (syphilis, nasal vestibulitis, tuberculosis), systemic disease (Wegener's granulomatosis, sarcoidosis, midline lethal granuloma, rhinoscleroma) and neoplasm (inverted papilloma, hemangioma, squamous cell carcinoma, lymphoma). In view of the appearance of the mass and the age of patient, our initial differential diagnoses were more focused on neoplasm, infectious and systemic causes. However, a series of laboratory test (rheumatoid factor test, antinuclear antibody test, rapid plasma reagin test, biopsy for acid fast bacillus and tuberculosis culture) and computed tomography of paranasal sinus showed inconclusive diagnosis until a repeated biopsy of 
Table 1 Comparison of different location of xanthogranulomatous inflammation and intervention outcome $(1,3,4,5,6)$

\begin{tabular}{|c|c|c|c|c|c|}
\hline Author & $\begin{array}{l}\text { Journal and year of } \\
\text { publication }\end{array}$ & Age & $\begin{array}{l}\text { Location of } \\
\text { xanthogranulomatous } \\
\text { inflammation }\end{array}$ & Treatment & Outcome \\
\hline Shashi Dhawan et al. & J. Crohns Colitis. 2011 (1) & 60 & Ascending colon & Right hemicolectomy & Well with 5 months follow-up \\
\hline Goyal S et al. & N Am J Med Sci. 2011 (3) & 40 & Kidney & Nephrectomy & Well with 6 months follow-up \\
\hline Kubota, Ket al. & J. Pancreas. 2017 (4) & 23 & Pancreas & Distal pancreatectomy & Well with 3 years follow-up \\
\hline Balasar M et al. & Urol Ann. 2017 (5) & 37 & Bladder & Partial cystectomy & Well with 1 year follow-up \\
\hline André Jacobsen et al. & Scand. J. Urol. 2019 (6) & 54 & Urethra & $\begin{array}{l}\text { Dexamethasone inject into } \\
\text { the tumour, followed by a } \\
\text { 4-week course of systemic } \\
\text { steroids }\end{array}$ & $\begin{array}{l}\text { Remission of tumour after } \\
2 \text { months of completed } \\
\text { treatment }\end{array}$ \\
\hline
\end{tabular}

the mass was conducted. Other reported visceral organ cases showed the similar diagnostic dilemma. In their cases, xanthogranulomatous inflammation could only be diagnosed by histopathological examination. The clinical and radiographic finding is non-specific and provides litthe value in the diagnosis of the disease [3-6].

The initial histopathological examination of the nasal cavity mass and nasopharynx revealed the presence of granulomatous inflammation. A granulomatous inflammation can be masked by the presence of infection, inflammatory reaction or neoplasm (e.g. lymphoma). With reference to our case, tuberculosis infection, Wegener granulomatosis and lymphoma were ruled out. In view of the uncertainty of the diagnosis, repeated biopsies were taken to arrive at a more conclusive, definitive diagnosis.

Documentation regarding its medical treatment is limited. Over the past decade, only a successful case of pharmacological therapy was reported in English literature review where a combination of intralesional and oral steroid was used in urethral xanthogranulomatosis which showed full remission [7]. However, in our case, empirical oral prednisolone over the course of 2 months did not show any response. Intralesional steroid was not an option as the mass was prone to bleeding upon manipulation.

In view of fail conservative and medical therapy, surgical excision was offered. In our patient, complete excision of the mass was hindered due to the excessive bleeding encountered intraoperatively. Thus, debulking surgery was performed. Difficulties in surgical dissection have been reported in xanthogranulomatous cholecystitis, where longer duration of cholecystectomy is needed if compared with other disorders, basically due to the adhesion and concomitant pathology [3].

Our case showed good surgical outcome that is comparable with the case reports mentioned earlier $[2,4-7]$. Table 1 showed the comparison of different locations of xanthogranulomatous inflammation and intervention outcome. At post-operative 6 months, despite the presence of residual nasal mass, the patient is asymptomatic without any recurrent epistaxis and nasal blockage. Having said that, our follow-up is short and longer follow-up is needed.

\section{Conclusion}

Xanthogranulomatous inflammation in the nasal cavity and nasopharynx can be easily confused with other nasal masses. A histopathology examination is crucial to establish the diagnosis. A repeated biopsy is needed if previous biopsy result is inconclusive. Surgical excision is recommended for symptomatic relief. The effectiveness of medical treatment is still unclear. A long-term follow-up is mandatory.

\section{Acknowledgements \\ None}

Authors' contributions

WJB and KSV conceived the original idea. This was also discussed with IPT. Eventually, all the authors discussed and agreed with the focus and ideas of this paper. The main text of the paper was written by WJB and edited by KSV and IPT. All authors revised the manuscript and contributed equally.

\section{Funding}

No funding is involved in this paper.

Availability of data and materials No applicable

\section{Declarations}

Ethics approval and consent to participate

Not applicable. Reference number is not available.

Consent for publication

Written informed consent was obtained from the patient.

\section{Competing interests}

The authors declare that they have no known competing financial interests or personal relationships that could have appeared to influence the work reported in this paper.

Received: 8 December 2021 Accepted: 31 December 2021 Published online: 24 January 2022 


\section{References}

1. Bourm KS, Menias CO, Ali K, Alhalabi K, Elsayes KM (2017) Spectrum of xanthogranulomatous processes in the abdomen and pelvis: a pictorial review of infectious, inflammatory, and proliferative responses. AJR Am J Roentgenol 208(3):475-484

2. Dhawan S, Jain D, Kalhan SK (2011) Xanthogranulomatous inflammation of ascending colon with mucosal involvement: report of a first case. J Crohns Colitis 5(3):245-248

3. Han SH, Chen YL (2012) Diagnosis and treatment of xanthogranulomatous cholecystitis: a report of 39 cases. Cell Biochem Biophys 64(2):131-135

4. Goyal S, Gupta M, Goyal R (2011) Xanthogranulomatous pyelonephritis: a rare entity. N Am J Med Sci 3:249-250

5. Kubota K, Nakayama A, Masuo H, Takasu K, Karasawa F, Takeuch N (2017) Multidisciplinary approach for progressive xanthogranulomatous pancreatitis: case report and review of the literature. J Pancreas 18(6):487-494

6. Balasar M, Sönmez MG, Oltulu P, Kandemir A, Kılı M, Gürbüz R (2017) Complete treatment with partial cystectomy in giant xanthogranulomatous cystitis case imitating bladder tumor. Urol Ann 9(2):204-207

7. Jacobsen A, Joensen UN, Jensen PH (2019) A case of xanthogranulomatous inflammation of the urethra: treatment with a steroid-based nonsurgical approach. Scand J Urol 53(4):267-268

\section{Publisher's Note}

Springer Nature remains neutral with regard to jurisdictional claims in published maps and institutional affiliations.

\section{Submit your manuscript to a SpringerOpen ${ }^{\odot}$ journal and benefit from:}

- Convenient online submission

- Rigorous peer review

- Open access: articles freely available online

- High visibility within the field

- Retaining the copyright to your article

Submit your next manuscript at $\boldsymbol{\nabla}$ springeropen.com 